\title{
Tumorigenesis: cell defense against hypoxia?
}

\author{
Nafiseh Pakravan \\ Department of Pathobiology, Medical School, Alborz University of Medical Sciences, Karaj, Iran
}

\begin{abstract}
Microenvironmental elements can directly contribute to the induction and the maintenance of tumor. Oxygen is the main element in the cell microenvironment and hypoxia can affect the process of tumorigenesis. In response to hypoxia, cells change their pattern and characteristics. These changes suggest that it is not just adaptation, but some sort of cell defense against hypoxia. If hypoxia is corrected, then cell defense mechanisms are interrupted. An examination of the process of tumorigenesis helps to design better therapeutic strategies.A systematic review of the English literature was conducted by searching PubMed, Google Scholar, and ISI Web databases for studies on changes that defend and help cells to live in a hypoxic microenvironment. Cells respond to hypoxia by de-differentiation and an increase in heat shock proteins. Angiogenesis and deviation of inflammatory response in favor of hypoxic cell survival also defend and save the oxygen-starved cells from death. Finally, anti-angiogenic therapies and more hypoxia enhance metastasis, as tumors with low oxygen concentration are more malignant than tumors with high oxygen concentration. All these enable cells to migrate away from low oxygen areas and seek a more conducive microenvironment. Therapies that make the microenvironment more hypoxic need to be revised. This has been done for antiangiogenic therapies, previously considered to be anti-tumor approaches. Effective therapies may be correcting therapies which direct the tumor microenvironment towards natural physical/chemical condition. Correcting therapies either bring back tumor cells to a normal form (correct tumor cells) or help the immune system to eradicate tumor cells which can not be corrected.
\end{abstract}

Correspondence: Nafiseh Pakravan, Department of Pathobiology, Medical School, Alborz University of Medical Sciences, Karaj, Iran.

Tel: +98.263 .4314400 - Fax: +98.263 .4319188 .

E-mail: nafiseh.pakravan@gmail.com

Key words: tumorigenesis/metastasis, differentiation, heat shock proteins, hypoxia, inflammation.

Received for publication: 11 January 2013.

Revision received: 2 March 2013.

Accepted for publication: 2 April 2013.

This work is licensed under a Creative Commons Attribution NonCommercial 3.0 License (CC BY-NC 3.0).

CCopyright N. Pakravan, 2012

Licensee PAGEPress, Italy

Oncology Reviews 2013; 6:e1

doi:10.4081/oncol.2013.e1

\section{Introduction}

Oxygen is a vital element required for cell survival and growth. Different levels of oxygen characterize healthy tissues, ranging from $150 \mathrm{mmHg}$ in the lung to $40-100 \mathrm{mmHg}$ in the circulation and $4-20 \mathrm{mmHg}$ in the tissues. ${ }^{1,2}$ Furthermore, the oxygen within a tissue is also variable and is proportional to the distance from the end of the nearest capillary; ${ }^{3}$ this is known as physiological hypoxia. On the other hand, pathological hypoxia develops as an aberrant status when tissue is damaged or degenerated. Pathological hypoxia creates a unique microenvironment, conditioning the phenotype and function of every cell type and delivering important signals to promote angiogenesis for improving oxygen delivery, activate an alternative energy source, and recruit effector leukocytes to deal with the disease. Oxygen tension in chronically hypoxic regions is less than $5 \mathrm{mmHg} .{ }^{4,5}$ Examples of pathological hypoxia include malignant solid tumors, inflammatory lesions, ischemic tissues, healing wounds, and obstructive pulmonary diseases. ${ }^{6}$

Adaptation to hypoxia is an essential homeostatic mechanism. Mammals are equipped with oxygen-sensing mechanisms that help them adapt quickly to hypoxia. If an inadequate supply of oxygen persists, additional mechanisms are activated to restore oxygenation or help the body adapt to hypoxia. These changes will be discussed with regard to tumor. Tumor cell adaptations to hypoxia mainly depend on the transcription factor hypoxia-inducible factor (HIF) which is activated in hypoxic conditions and is inactive when oxygen is abundant. ${ }^{7,8} \mathrm{HIF}$ regulates genes of intracellular proteins and receptors, and activation of HIF leads to changes in tumor cell function. These changes include de-differentiation, angiogenesis, invasion and metastasis formation,,${ }^{9,10}$ and the glucose and energy metabolism. ${ }^{11}$

It is generally accepted that cancer is not merely an accumulation of genetic mutations that lead to unharnessed cell proliferation, but that it is mainly dependent up on the surrounding microenvironment. Since the cell microenvironment has a critical role in cancer formation and progression, this paper discusses the role of hypoxia as the main factor in tumourigenesis.

\section{Hypoxia, inflammation and tumorigenesis}

Inflammation is a defensive reaction of living tissue to injury. Clinical evidence indicates that hypoxia promotes inflammation. In a mutually exclusive fashion, hypoxia can promote inflammation and inflamed lesions are often severely hypoxic. Indeed, hypoxia can influence the tissue environment of the tissue, particularly by regulating oxygen-dependent gene expression. ${ }^{12}$ Cancer is a clinical condition in which hypoxia and inflammation interact at several points. 
As early as the $19^{\text {th }}$ century, cancers were understood to arise from sites of chronic inflammation, infection and chronic irritation. ${ }^{13,14}$ Characteristics of inflammation have been established even in tumors with no relationship to infection, such as breast tumors. Epidemiological studies are consistent with the notion that chronic inflammation predisposes to different types of cancer, suggesting a link between infections and inflammatory responses. ${ }^{13}$ It is generally accepted that tumors and sites of inflammation, such as wounds, have many similarities in common. Tumors can be thought of as wounds that will not heal. ${ }^{15} \mathrm{~A}$ variety of inflammatory mediators, such as interleukin (IL)-1, IL-6, tumor necrosis factor- $\alpha$, vascular endothelia growth factor (VEGF), and eicosanoids affect tumor progression. ${ }^{16}$

At the site of tumor foci, inflammatory response recruits leukocytes through the production of various cytokines and chemokines, including VEGF and CXCR4, involved in angiogenesis and metstasis. ${ }^{17}$ Hypoxia via HIF affects the performance of innate immunity. The tumor-recruited leukocyte population is heterogeneous and consists, in part, of tumor-associated neutrophils (TANs) and tumorassociated macrophages (TAMs) that acquire N2 and M2 phenotypes under the hypoxic environmental conditions of the tumor, respectively. TANs with N2 phenotype have a pro-tumorigenic effect secreting pro-tumorigenic factors, promoting angiogenesis and suppressing immune responses. ${ }^{18-20}$ Recent studies have also suggested a pro-metastatic role for TANs with N2 phenotype. There is evidence to suggest that a hypoxic environment gives N2 phenotype to TANs at the tumor foci, whereas neutrophils seeding in the lungs with a normoxic environment acquire a N1 phenotype and have anti-metastatic effects. ${ }^{21}$ In addition, hypoxia via HIF dramatically redirects differentiation of TAMs toward M2 in the tumor microenvironment. M2 macrophages produce cytokines and other mediators which favor tumor growth and progression. ${ }^{22,23}$

The hypoxic environment of the tumor influences adaptive immunity by stimulation of regulatory T-cell (Treg) activation and differentiation. ${ }^{2,24,25}$ In addition, hypoxia affects denderitic cell differentiation in the same way that naïve T cells favor T-helper 2 (Th2) response. ${ }^{12} \mathrm{Re}$ oxygenation of hypoxia-differentiated dendritic cells induces Thl and Th17 cell differentiation, restricting tumor growth and progression. ${ }^{26,27}$ In breast cancer, a high $\mathrm{CD} 4{ }^{+} / \mathrm{CD}^{+}$and Th2/Th1 ratio of tumor-infiltrating lymphocytes is indicative of poor prognosis. ${ }^{28} \mathrm{On}$ the other hand, TAMs are educated by Th2 $\mathrm{CD}^{+} \mathrm{T}$ cells to produce proangiogenic and pro-metastatic factors stimulating mammary cancer progression and metastasis. ${ }^{22}$ In addition, there is also evidence that many of the T-cell subsets found in solid tumors can aid tumor promotion, progression, or metastasis, including $\mathrm{CD} 8{ }^{+} \mathrm{T}$ cells, ${ }^{29}$ interferon producing Th1 cells, ${ }^{30} \mathrm{Th} 2$ cells $^{22,31}$ and Th17 cells. ${ }^{32,33}$ Nevertheless, natural killer cells are the only cells that, so far, have not shown a protumorigenic role. The determining factor that gives the same T-cell subset an anti-tumorigenic role in one cancer and a pro-tumorigenic role in another is for the most part not known.

Surprisingly, Treg cells, which are known to play a pro-tumorigenic role through suppression of anti-tumor immune responses, ${ }^{34}$ may also play an anti-tumorigenic role under certain circumstances by virtue of their ability to suppress tumor-promoting inflammation. ${ }^{35}$ All of the evidence mentioned above indicates that there is hypoxic microenvironment present in chronic inflammation and cancer, and that, therefore, they are closely linked. Angiogenesis is codependent with chronic inflammation and cancer; 36,37 this is a process dictated by a hypoxic microenvironment to overcome the starvation of oxygen and is observed in both conditions. Inflammatory cells, mainly M2 cells, and immunological mediators such as VEGF, chemokines, eicosanoids, and reactive oxygen, are involved in angiogenesis. ${ }^{16}$

\section{Hypoxia and angiogenesis}

The induction of new blood vessels by solid tumors was first recognized by Virchow nearly 150 years ago, but tumor angiogenesis is frequently linked to the name of Dr. Judah Folkman who proposed that the growth of all solid tumors is dependent on angiogenesis. ${ }^{38}$ It is an important event in various biological processes, including embryonic vascular development and organ regeneration. Angiogenesis is also observed in many pathological conditions, such as inflammation, wound healing, and tumor growth. Nevertheless, tumor vasculature differs in many aspects from the vasculature of normal organs. ${ }^{39}$

Hypoxia is well known for its role in the induction of angiogenesis in hypoxic zones and for its essential involvement in further tumor development. ${ }^{40} \mathrm{HIF}$ regulates genes that are key players in cancer development. They include many angiogenic mediators, such as VEGF, genes involved in cellular matrix metabolism (urokinase-type plasminogen activator receptor, matrix metalloproteinases-2 MMP2) and glycolysis (phosphoglycerate kinase-1 and lactate dehydrogenase ${ }^{41}$ ). Haplode letion of prolylhydroxylases, which hydroxylate and facilitate HIF destruction, in mice leads to vascular normalization, as defined by more sharply demarcated boundaries and branching points of tumor vessels ${ }^{42}$ and tumor oxygenation. Vascular normalization is due to attenuation of tumor-vessel leakiness and vascular distortion and improvement of tumor-vessel architecture. This change reduces tumor invasiveness and the risk of metastasis. ${ }^{43}$ However, other reports indicate that therapeutic strategies targeting the inhibition of neoangiogenesis lead to HIF activation and promotion of metastasis formation. ${ }^{44,45}$ This suggests that oxygen-deprivation and prolonged enforced oxygen-deprivation of tumors by anti-angiogenic drugs can increase migratory activity and metastatic potential. Thus, therapeutic strategy in cancer treatment targeting inhibition of tumor vascularization leads to a sustained oxygen deprivation and may change the behavior of tumor cells so that they are either beneficial for the treatment, or can even worsen it. Therefore, the degree of hypoxia after treatment at the tumor foci can have a direct effect on the efficiency of the therapeutic strategy. Since the degree of hypoxia also affects cell differentiation status, some therapeutic strategies target this status in tumor cells.

\section{Hypoxia, differentiation and tumorigenesis}

Cell differentiation shows the degree of cell maturity. Fully differentiated (fully mature) cells have the same form and function as their parent cells and proliferate very slowly, if at all. In contrast, immature cells do not resemble their parents, are poorly differentiated, and are able to proliferate at a higher rate. Most cancer cells are less differentiated and less mature than normal cells and, therefore, proliferate readily.

The degree of cell differentiation is regulated by gene expression. Therefore, manipulation of gene expression can change the degree of differentiation. A number of natural compounds can induce differentiation in cancerous cells, decrease the proliferation rate of cancerous cells, and make them display fewer malignant characteristics. ${ }^{46}$

There is increasing evidence to suggest that hypoxia is the potent inhibitor of tumor cell differentiation and thus plays a key role in the maintenance of cancer stem cells, likely via the evolution of the tumor stromal microenvironment. ${ }^{47}$

The main hypoxia-activated pathway in cancer cell differentiation is mediated by HIF. HIF has been shown to inhibit or even reverse cell differentiation. ${ }^{48}$ De-differentiation was induced by hypoxia and the cells exhibited downregulation of p53 gene expression. ${ }^{49-53}$ Overexpression 
of HIF has been shown to induce epithelial-mesenchymal transition (EMT), and metastatic phenotypic and migration activity change both in vitro and in vivo. Inversely, the process of EMT can be reversed by repression of HIF expression in hypoxic cells. HIF can regulate transcription factors, e.g. Snail, LOX or TWIST, involved in EMT. ${ }^{54-56}$ Hypoxia can also act via activation of other key regulatory pathways in stem/progenitor cells, such as transforming-growth factor- $\beta$, CXCR4 and its ligand CXCL12 or stromal cell-derived factor-1, and erythropoietin-erythropoietin receptor pathway. ${ }^{47}$

The advantage of arresting tumorigenic cells in their undifferentiated state by hypoxia is that this makes immature stem cells more resistant to therapy compared to differentiated and partially differentiated stem cells, and also maintains their clonogenic/tumorigenic potential. ${ }^{57}$ Presumably, de-differentiation occurs as a defensive mechanism to protect hypoxic cells against further hypoxia. Another important defense mechanism activated in response to hypoxia is increased expression of heat shock proteins (HSPs).

\section{Hypoxia, cellular heat shock protein level and tumorigenesis}

Heat shock proteins are highly conserved molecules, some of which are induced by cellular stresses, including hypoxia and oxidative damage ${ }^{58} \mathrm{HSP}$ activation is critical to adaptation to hypoxia. There is a regulatory link between the cell oxygen sensors and heat shock pathways. Expression of inducible HSPs is regulated by heat shock factors (HSF), in particular HSF1 ${ }^{59,60} \mathrm{HSF}$ transcription is necessary for full HSP induction during hypoxia. Some reports have suggested that stresses associated with energy deprivation can activate HIF-1. ${ }^{59-61}$ Expression of HSF is up-regulated during hypoxia due to direct binding by HIF-1 to HSF promoter. ${ }^{62}$ Increased levels of HSPs have been detected in many solid tumors. The HIF-1-dependent increase in HSPs provide a transient protection from stress, as reduced production of HSPs decreases viability of multiple human cancer cell lines, but had no effect on normal cells, suggesting that HSF-1 relieves cancer cells from the cellular stresses. ${ }^{63}$ HSPs provide a transient protection from stress acting as chaperones by regulation of protein folding to ensure correct conformation and translocation. ${ }^{58}$ It may explain the ability of malignant cells to maintain protein homoeostasis even in the hostile hypoxic microenvironment of the tumor. Accordingly, HSPs may account for the ability of tumor cells to tolerate genetic alterations that would otherwise be fatal. ${ }^{64}$ Presumably, HSPs support tumorigenesis by orchestrating a broad network of cellular pathways that counteract apoptosis and promote tumor cell survival, autonomous cell proliferation, and metastasis, even in hypoxic, acidotic, and growth factor-deprived conditions. ${ }^{63,65,66}$ The cytoprotective function of HSPs is also explained by their anti-apoptotic function. The anti-apoptotic properties of HSPs can partly be explained by their ability to interact with different proteins of the apoptotic process at distinct key points, thereby directly blocking programmed cell death machinery/mechanisms. In addition, it has been reported that the increased HSP expression in tumor cells is due to loss of p53 function. This HSP action in tumor cells can account for inhibition of apoptosis. ${ }^{62}$

Apoptosis and differentiation share many common features. Many recent reports implicate HSPs in the differentiation process. Accordingly, HSPs are induced during oncogenesis, as well as at specific stages of development and differentiation. ${ }^{67}$ This indicates that HSPs not only protect cancerous cells against a hostile hypoxic microenvironment but also act in orchestration with de-differentiation of cancerous cells in the hypoxic condition. Presumably, HSPs support tumorigenesis by orchestrating a broad network of cellular pathways that counter- act apoptosis and promote tumor cell survival, autonomous cell proliferation, and metastasis, even in hypoxic, acidotic, and growth factordeprived conditions. . $^{63,65,66}$

\section{Hypoxia and metastasis}

Metastasis is a process in which diverse interactions between cancer cells and their microenvironment allow the cells to populate and flourish in new tissue habitats. This follows organ dysfunction and death. Angiogenesis, and the proliferation of a network of blood vessels that penetrates into cancerous tissue supplying nutrients and oxygen, trigger metastasis. ${ }^{68}$ Therapeutics strategies that impede angiogenesis have been proposed to combat malignancy. ${ }^{69}$ However, some reports have suggested that angiogenic inhibitors may sometimes induce a more invasive type of tumor and increase risk of metastasis. ${ }^{44,45}$ It is thought that hypoxia enhances metastases, as tumors with low oxygen concentration are more malignant than tumors with high oxygen concentration..$^{70}$ In vitro experiments have demonstrated that exposure to hypoxia increases migration, invasion and adhesion of cancerous cells. Inversely, RNA interference in silencing HIF-1 resulted in a marked decrease in cancerous cell migration, invasion and adhesion. Analysis of a number of genes which regulate cancer invasion and metabolism, such as CXCR4, angiopoietin-related protein, and pyruvate dehydrogenase kinase 1, revealed that they are also activated by hypoxia in cancerous cells. ${ }^{71}$ Accordingly, in vivo experiments have also demonstrated that hypoxic conditions increase the expression of angiogenic factors, including VEGF, angiogenin, and IL-8, and molecules involved in motility and adhesion of cancerous cells, including autocrine motility factor, MMPs, and selectin-ligands, leading to the increase in angiogenesis, motility, and adhesion of cancerous cells through which they invade the blood circulation. ${ }^{70}$ Accordingly, it has been revealed that CXCR4 is activated by the lack of oxygen and this activation makes tumor cells migrate and home in on a specific set of organs. Indeed, combined expression of HIF-1, CXCR4, and VEGF strongly correlates with lymph node metastasis and distant metastasis. ${ }^{72,73}$

Altogether, it seems that the proteins encoded by these HIF-responsive genes have a variety of functions and aid the hypoxic cells by either increasing tissue oxygenation, e.g. VEGF (which stimulates the outgrowth of new blood vessels) or by enhancing cellular glucose uptake and metabolism to allow energy generation when oxygen is scarce. All of these enable the cells to migrate away from low oxygen areas and home to specific, distant organs. ${ }^{74}$

\section{Conclusions}

In general, the term adaptation is used for the changes imposed by hypoxia. I propose defense instead of adapt, as I believe this describes the situation better, since events ending to tumorigenesis are not some sort of diplomatic negotiation but a battle. The term defense shows that a harsh disturbance, i.e. hypoxia, has been imposed on the microenvironment and cells have to respond by undergoing change. The changes made by defensive mechanisms are not optimal for cells but they are committed to making them. Our understanding of the process of tumorigenesis helps us design better therapeutic strategies.

Hypoxic cells defend against or respond to hypoxia by angiogenesis and by activating the expression of genes involved in better tissue oxygenation. This causes the cells to escape necrosis and apoptosis and survive. However, if the situation worsens, severe hypoxia, prolonged hypoxia, or anoxia may initiate a cascade of events that leads to apop- 
totic cell death. Severe hypoxia, prolonged hypoxia, or anoxia may initiate apoptosis, whereas cells can often defend themselves against acute and mild hypoxia and survive. The severity of hypoxia determines whether cells can survive or enter apoptosis. ${ }^{75}$ This is well illustrated by the experiments performed by Butterworth and co-workers who generated a human prostate cancer cell subline by extended oxygen-deprivation. ${ }^{76}$ In other words, it seems that cells become cancerous in order to defend themselves against environmental insult, mainly hypoxia and hypoxia, increasing intrinsic tumorigenic potential. ${ }^{47}$ This is shown more clearly by an increase in HSPs and de-differentiation under hypoxic conditions. The starving cells de-differentiate as an economically compensatory defense mechanism against hypoxia. Inversely, it has been shown that differentiation status can be improved and tumor arrest can be induced if exposed to higher oxygen concentrations. ${ }^{51,77}$ In addition, experiments demonstrating vascular normalization and tumor oxygenation may be beneficial for treatment. It seems that the last line of cell immune response is performed by metastasis, as demonstrated by the notion that starving tumor cells of oxygen is one trigger for metastasis. ${ }^{78,79}$ It seems that the starving cells travel and home into an environment that is more conducive to live in. Finally, under severe hypoxia or anoxia, the cells submit and commit suicide, as observed by apoptosis. Thus, effective therapeutic strategies may be those that correct the physica//chemical condition of the tumor microenvironment as this affects tumor cells, stromal cells of the tumor site, and the immune system. Correcting therapies which correct the physical/chemical condition of the tumor microenvironment may either help tumor cells to return to normal form (correct tumor cells) and/or help the immune system to eradicate tumor cells which do not correct or do not return to normal form (uncorrected cells). Based on the plasticity that protects cells from damage caused by hypoxia and enables cells to de-differentiate, ${ }^{80}$ it may be possible to promote cancerous cells to its differentiated state by correcting therapy. The aim of this approach is to reprogram cancerous cells towards a normal state; this may depend on tissue type, as oxygen tension is different in different tissues. ${ }^{77,80,81}$

Notably, tumorigenesis does not happen suddenly, but is a process influenced by elements such as timing and target tissue. Accordingly, a simple oxygenation will not correct the microenvironment and so correct tumor cells. This is the same for any therapeutic agent. Any medication is administered according to a program, and correcting therapy also follows this rule. A model of malignancy is pregnancy and the fetus is regarded as a pseudomalignancy. The fetus is delivered/rejected after differentiation. As in embryogenesis, during which a gradient of hypoxia is involved in differentiation, ${ }^{82-84}$ it has been suggested that correcting therapy in a programmed approach re-differentiates cancerous cells. Administration of correcting therapy should be set up in a programmed way, and may be administered with graded exposure or according to a planned gradient. It may even vary depending on the target tissue or may even complement it with current therapies.

In this review, correcting therapies using oxygen has been proposed because oxygen tension affects many aspects of the tumor microenvironment, such as acidity, which hampers current therapies. ${ }^{85}$ The idea of correcting therapy is still in its infancy and its application needs to be developed, and, as with other therapeutics approaches, dose and planning of correcting therapies using oxygen need to be set up.

\section{References}

1. Semenza GL. Hypoxia-inducible factor 1: oxygen homeostasis and disease pathophysiology. Trends Mol Med 2001;7:345-50.

2. Sitkovsky M, Lukashev D. Regulation of immune cells by local-tis- sue oxygen tension: HIF1 alpha and adenosine receptors. Nat Rev Immunol 2005;5:712-21.

3. Evans SM, Hahn SM, Magarelli DP, Koch CJ. Hypoxic heterogeneity in human tumors: EF5 binding, vasculature, necrosis, and proliferation. Am J Clin Oncol 2001;24:467-72.

4. Kallinowski F, Zander R, Hoeckel M, Vaupel P. Tumor tissue oxygenation as evaluated by computerized-p02-histography. Int $\mathrm{J}$ Radiat Oncol Biol Phys 1990;19:953-61.

5. Vaupel P, Schlenger K, Knoop C, Hockel M. Oxygenation of human tumors: evaluation of tissue oxygen distribution in breast cancers by computerized 02 tension measurements. Cancer Res 1991;51:3316-22.

6. Bosco MC, Puppo M, Blengio F, et al. Monocytes and dendritic cells in a hypoxic environment: Spotlights on chemotaxis and migration. Immunobiology 2008;213:733-49.

7. Semenza GL. Life with oxygen. Science 2007;318:62-4.

8. Kaelin WG Jr, Ratcliffe PJ. Oxygen sensing by metazoans: the central role of the HIF hydroxylase pathway. Mol Cell 2008;30:393-402.

9. Gordan JD, Simon MC. Hypoxia-inducible factors: central regulators of the tumor phenotype. Curr Opin Genet Dev 2007;17:71-7.

10. Semenza GL. Targeting HIF-1 for cancer therapy. Nat Rev Cancer 2003;3:721-32.

11. Semenza GL. Regulation of cancer cell metabolism by hypoxiainducible factor 1. Semin Cancer Biol 2009;19:12-6.

12. Eltzschig HK, Carmeliet P. Hypoxia and inflammation. N Engl J Med 2011;364:656-65.

13. Balkwill F, Mantovani A. Inflammation and cancer: back to Virchow? Lancet 2001;357:539-45.

14. Nyberg P, Salo T, Kalluri R. Tumor microenvironment and angiogenesis. Front Biosci 2008;13:6537-53.

15. Dvorak HF. Tumors: wounds that do not heal. Similarities between tumor stroma generation and wound healing. N Engl J Med 1986;315:1650-9.

16. Wu P. Cancer related inflammation and tumor angiogenesis, tumor angiogenesis. In: Ran S, ed. Tumor angiogenesis. Rijeka: InTech; 2012. Available from: http://www.intechopen.com/books/tumorangiogenesis/cancer-related-inflammation-and-tumor-angiogenesis

17. Coussens LM, Werb Z. Inflammation and cancer. Nature 2002;420:860-7.

18. Pekarek LA, Starr BA, Toledano AY, Schreiber H. Inhibition of tumor growth by elimination of granulocytes. J Exp Med 1995;181:435-40.

19. Shojaei F, Singh M, Thompson JD, Ferrara N. Role of Bv8 in neutrophil dependent angiogenesis in a transgenic model of cancer progression. Proc Natl Acad Sci U S A 2008;105:2640-5.

20. Youn JI, Nagaraj S, Collazo M, Gabrilovich DI. Subsets of myeloidderived suppressor cells in tumor-bearing mice. J Immunol 2008;181:5791-802.

21. Granot Z, Henke E, Comen EA, et al. Tumor entrained neutrophils inhibit seeding in the premetastatic lung. Cancer Cell 2011;20:30014.

22. DeNardo DG, Barreto JB, Andreu P, et al. CD4(+) T cells regulate pulmonary metastasis of mammary carcinomas by enhancing protumor properties of macrophages. Cancer Cell 2009;16:91-102.

23. Mantovani A, Sozzani S, Locati M, et al. Macrophage polarization: tumor-associated macrophages as a paradigm for polarized M2 mononuclear phagocytes. Trends Immunol 2002;23:549-55.

24. Ben-Shoshan J, Maysel-Auslender S, Mor A, et al. Hypoxia controls CD4+CD25+ regulatory T-cell homeostasis via hypoxia-inducible factor-1alpha. Eur J Immunol 2008;38:2412-8.

25. Kojima H, Gu H, Nomura S, et al. Abnormal B lymphocyte development and autoimmunity in hypoxia-inducible factor lalpha-deficient chimeric mice. Proc Natl Acad Sci U S A 2002;99:2170-4.

26. Ikejiri A, Nagai S, Goda N, et al. Dynamic regulation of Th17 differ- 
entiation by oxygen concentrations. Int Immunol 2012;24:137-46.

27. Wang Q, Liu C, Zhu F, et al. Reoxygenation of hypoxia-differentiated dentritic cells induces Th1 and Th17 cell differentiation. Mol Immunol 2012;47:922-31.

28. Kohrt HE, Nouri N, Nowels K, et al. Profile of immune cells in axillary lymph nodes predicts disease-free survival in breast cancer. PLoS Med 2005;2:e284.

29. Roberts SJ, Ng BY, Filler RB, et al. Characterizing tumor-promoting $\mathrm{T}$ cells in chemically induced cutaneous carcinogenesis. Proc Natl Acad Sci U S A 2007;104:6770-5.

30. Hanada T, Kobayashi T, Chinen T, et al. IFNgamma-dependent, spontaneous development of colorectal carcinomas in SOCS1-deficient mice. J Exp Med 2006;203:1391-7.

31. Aspord C, Pedroza-Gonzalez A, Gallegos M, et al. Breast cancer instructs dendritic cells to prime interleukin 13-secreting CD4+ T cells that facilitate tumor development. J Exp Med 2007;204:103747.

32. Langowski JL, Kastelein RA, Oft M. Swords into plowshares: IL-23 repurposes tumor immune surveillance. Trends Immunol 2007;228 207-12.

33. Wang L, Yi T, Kortylewski M, et al. IL-17 can promote tumor growth through an IL-6-Stat3 signaling pathway. J Exp Med 2009;206:145764.

34. Gallimore AM, Simon AK. Positive and negative influences of regulatory T cells on tumour immunity. Oncogene 2008;27:5886-93.

35. Erdman SE, Sohn JJ, Rao VP, et al. CD4+CD25+ regulatory lymphocytes induce regression of intestinal tumors in ApcMin/+ mice. Cancer Res 2005;65:3998-4004.

36. Alexandrescu DT, Riordan NH, Ichim TE, et al. On the missing link between inflammation and cancer. Dermatol Online J 2011;17:10.

37. Kobayashi H, Lin PC. Angiogenesis links chronic inflammation with cancer. Methods Mol Biol 2009;511:185-91.

38. Folkman J. Tumor angiogenesis: therapeutic implications. N Engl J Med 1971;285:1182-6.

39. Fierro IM. Angiogenesis and lipoxins. Prostaglandins Leukot Essent Fatty Acids 2005;73:271-5

40. Sermeus A, Michiels C. Reciprocal influence of the p53 and the hypoxic pathways. Cell Death Dis 2011;2:e164.

41. Weidemann A, Johnson RS. Biology of HIF-1alpha. Cell Death Differ 2008;15:621-7.

42. Canning MT, Postovit LM, Clarke SH, Graham CH. Oxygen-mediated regulation of gelatinase and tissue inhibitor of metalloproteinases-1 expression by invasive cells. Exp Cell Res 2001;267:8894.

43. Osinsky SP, Ganusevich II, Bubnovskaya LN, et al. Hypoxia level and matrix metalloproteinases- 2 and -9 activity in Lewis lung carcinoma: correlation with metastasis. Exp 0ncol 2005;27:202-5.

44. Ebos JM, Lee CR, Cruz-Munoz W, et al. Accelerated metastasis after short-term treatment with a potent inhibitor of tumor angiogenesis. Cancer Cell 2009;15:232-9.

45. Loges S, Mazzone M, Hohensinner P, Carmeliet, P. Silencing or fueling metastasis with VEGF inhibitors: antiangiogenesis revisited. Cancer Cell 2009;15:167-70.

46. Gullett NP, Ruhul Amin AR, Bayraktar S, et al. Cancer prevention with natural compounds. Semin Oncol 2010 37:258-81.

47. Kim Y, Lin Q, Glazer PM, Yun Z. Hypoxic tumor microenvironment and cancer cell differentiation. Curr Mol Med 2009;9:425-34.

48. Axelson H, Fredlund E, Ovenberger M, et al. Hypoxia-induced dedifferentiation of tumor cells - a mechanism behind heterogeneity and aggressiveness of solid tumors. Semin Cell Dev Biol 2005; 16:554-63.

49. Jee MK, Kim JH, Han YM, et al. DHP-derivative and low oxygen tension effectively induces human adipose stromal cell programming. PLoS One 2010;5:e9026.
50. Jing D, Wobus M, Poitz DM, et al. Oxygen tension plays a critical role in the hematopoietic microenvironment in vitro. Haematologica 2012;97:331-9.

51. Liu W, Guo M, Xu YB, et al. Induction of tumor arrest and differentiation with prolonged survival by intermittent hypoxia in a mouse model of acute myeloid leukemia. Blood 2006;107:698-707.

52. Mannello F, Medda V, Tonti GA. Hypoxia and neural stem cells: from invertebrates to brain cancer stem cells. Int J Dev Biol 2001;55:56981.

53. Schnitzer SE, Schmid T, Zhou J, Brüne B. Hypoxia and HIF-1alpha protect A549 cells from drug-induced apoptosis. Cell Death Differ 2006;13:1611-3.

54. Krishnamachary B, Zagzag D, Nagasawa H, et al. Hypoxiainducible factor-1-dependent repression of E-cadherin in von Hippel-Lindau tumor suppressor-null renal carcinoma mediated by TCF3, ZFHX1A, and ZFHX1B. Cancer Res 2006;66:2725-31.

55. Evans AJ, Russell RC, Roche 0, et al. VHL promotes E2 box-dependent E-cadherin transcription by HIF-mediated regulation of SIP1 and Snail. Mol Cell Biol 2007;27:157-69.

56. Yang MH, Wu MZ, Chiou SH, et al. Direct regulation of TWIST by HIF-1alpha promotes metastasis. Nat Cell Biol 2008;10:295-305.

57. Wagemaker G. Heterogeneity of radiation sensitivity of hemopoietic stem cell subsets. Stem Cells 1995;13:257-60.

58. Young JC, Agashe VR, Siegers K, Hartl FU. Pathways of chaperonemediated protein folding in the cytosol. Nat Rev 2004;5:781-91.

59. Xiao X, Zuo X, Davis AA, et al. HSF1 is required for extra-embryonic development, postnatal growth and protection during inflammatory responses in mice. EMBO J 1999;18:5943-52.

60. Ruscher K, Isaev N, Trendelenburg G, et al. Induction of hypoxia inducible factor 1 by oxygen glucose deprivation is attenuated by hypoxic preconditioning in rat cultured neurons. Neurosci Lett 1998;254:117-20.

61. Eickelberg 0, Seebach F, Riordan M, et al. Functional activation of heat shock factor and hypoxia-inducible factor in the kidney. J Am Soc Nephrol 2002;13:2094-101.

62. Baird NA, Turnbull DW, Johnson EA. Induction of the heat shock pathway during hypoxia requires regulation of heat shock factor by hypoxia-inducible factor-1. J Biol Chem 2006;281:38675-81.

63. Dai C, Whitesell L, Rogers AB, Lindquist S. Heat shock factor 1 is a powerful multifaceted modifier of carcinogenesis. Cell 2007;130:1005-18.

64. Bagatell R. Altered Hsp90 function in cancer: a unique therapeutic opportunity. Mol Cancer Ther 2004;3:1021-30.

65. Whitesell L, Lindquist SL. HSP90 and the chaperoning of cancer. Nat Rev Cancer 2005;5:761-72.

66. Birch-Machin I, Gao S, Huen D, et al. Genomic analysis of heatshock factor targets in Drosophila. Genome Biol 2005;6:R63.

67. Parcellier A, Gurbuxani S, Schmitt E, et al. Heat shock proteins, cellular chaperones that modulate mitochondrial cell death pathways. Biochem Biophys Res Commun 2003;304:505-12.

68. Zetter BR. Angiogenesis and tumor metastasis. Ann Rev Med 1998;49:407-24

69. Siemann DW, Chaplin DJ, Horsman MR. Vascular-targeting therapies for treatment of malignant disease. Cancer 2004;100:241-9.

70. Masanobu K. HIF-1 and metastasis. Biotherapy (Tokyo) 2004;18:389-96

71. Victor N, Ivy A, Jiang BH, Agani FH. Involvement of HIF-1 in invasion of Mum2B uveal melanoma cells. Clin Exp Metastasis 2006;23:87-96.

72. Ishikawa T, Nakashiro K, Klosek SK, et al. Hypoxia enhances CXCR4 expression by activating HIF-1 in oral squamous cell carcinoma. Oncol Rep 2009;21:707-12.

73. Wu Y, Jin M, Xu H, et al. Clinicopathologic significance of HIF-1 $\alpha$, CXCR4, and VEGF expression in colon cancer. Clin Dev Immuno 
2010:537531.

74. Bernards R. Cancer: cues for migration. Nature 2003;425:247-8.

75. Greijer AE, van der Wall E. The role of hypoxia inducible factor 1 (HIF-1) in hypoxia induced apoptosis. J Clin Pathol 2004;57:100914.

76. Butterworth KT, McCarthy HO, Devlin A, et al. Hypoxia selects for androgen independent $\mathrm{LNCaP}$ cells with a more malignant genoand phenotype. Int J Cancer 2008;123:760-8.

77. Ivanovic Z. Hypoxia or in situ normoxia: the stem cell paradigm. J Cell Physiol 2009;219:271-5.

78. Bottaro DP, Liotta LA. Cancer: out of air is not out of action. Nature 2003;423:593-5.

79. Pennacchietti S, Michieli P, Galluzzo M, et al. Hypoxia promotes invasive growth by transcriptional activation of the met protooncogene. Cancer Cell 2003;3:347-61.

80. Das S, Foley N, Bryan K, et al. MicroRNA mediates DNA demethy- lation events triggered by retinoic acid during neuroblastoma cell differentiation. Cancer Res 2010;70:7874-81.

81. Lunt SJ, Chaudary N, Hill RP. The tumor microenvironment and metastatic disease. Clin Exp Metastasis 2009;26:19-34.

82. Stroka DM, Burkhardt T, Desbaillets I, et al. HIF-1 is expressed in normoxic tissue and displays an organ-specific regulation under systemic hypoxia. FASEB J 2001;15:2445-53.

83. Cejudo-Martin P, Johnson RS. A new notch in the HIF belt: how hypoxia impacts differentiation. Dev Cell 2005;9:575-6.

84. Gultice AD, Kulkarni-Datar K, Brown TL. Hypoxia-inducible factor 1 alpha (HIF1A) mediates distinct steps of rat trophoblast differentiation in gradient oxygen. Biol Reprod 2009;80:184-93.

85. Gillies RJ, Schornack PA, Secomb TW, Raghunand N. Causes and effects of heterogeneous perfusion in tumors. Neoplasia 1999;1:197-207. 